\title{
Existence and multiplicity for positive solutions of a system of higher-order multi-point boundary value problems
}

Johnny Henderson and Rodica Luca

\begin{abstract}
We investigate the existence and multiplicity of positive solutions of multi-point boundary value problems for systems of nonlinear higher-order ordinary differential equations.
\end{abstract}

Mathematics Subject Classification. 34B10, 34B18.

Keywords. higher-order differential system, multi-point boundary conditions, positive solutions.

\section{Introduction}

We consider the system of nonlinear higher-order ordinary differential equations

$$
\left\{\begin{array}{l}
u^{(n)}(t)+f(t, v(t))=0, \quad t \in(0, T), \quad n \in \mathbb{N}, \quad n \geq 2, \\
v^{(m)}(t)+g(t, u(t))=0, \quad t \in(0, T), \quad m \in \mathbb{N}, \quad m \geq 2,
\end{array}\right.
$$

with the multi-point boundary conditions

$$
\left\{\begin{array}{lll}
u(0)=u^{\prime}(0)=\cdots=u^{(n-2)}(0)=0, & u(T)=\sum_{i=1}^{p-2} a_{i} u\left(\xi_{i}\right), & p \in \mathbb{N}, \quad p \geq 3, \\
v(0)=v^{\prime}(0)=\cdots=v^{(m-2)}(0)=0, & v(T)=\sum_{i=1}^{q-2} b_{i} v\left(\eta_{i}\right), \quad q \in \mathbb{N}, \quad q \geq 3 .
\end{array}\right.
$$

Under sufficient assumptions on $f$ and $g$, we prove the existence and multiplicity of positive solutions of the above problem, by applying the fixed point index theory. By a positive solution of $(S)-(B C)$, we understand a pair of functions $(u, v) \in C^{n}([0, T]) \times C^{m}([0, T])$ satisfying $(S)$ and $(B C)$ with $u(t) \geq$ $0, v(t) \geq 0$ for all $t \in[0, T]$, and $\sup _{t \in[0, T]} u(t)>0, \sup _{t \in[0, T]} v(t)>0$. This problem is a generalization of the problem studied in [5], where $n=m=2$. In [16], the authors investigated the existence and multiplicity of positive solutions for system $(S)$ with $n=m=2$ and $T=1$ with the boundary conditions 
$u(0)=0, u(1)=\alpha u(\eta), v(0)=0, v(1)=\alpha v(\eta), \eta \in(0,1), 0<\alpha \eta<1$. We also mention the paper [14] where the authors used the fixed point index theory to prove the existence of positive solutions for the system $(S)$ with $f(t, v(t))$ and $g(t, u(t))$ replaced by $c(t) \widetilde{f}(u(t), v(t))$ and $d(t) \widetilde{g}(u(t)), v(t))$, respectively, and $(B C)$, where $\frac{1}{2} \leq \xi_{1}<\xi_{2}<\cdots<\xi_{p-2}<1, \frac{1}{2} \leq \eta_{1}<\eta_{2}<\cdots<\eta_{q-2}<1$, $(T=1)$. Some multi-point boundary value problems for systems of ordinary differential equations which involve positive eigenvalues were studied in recent years by Henderson, Luca, Ntouyas and Purnaras, by using the Guo-Krasnosel'skii fixed point theorem. In [3], the authors give sufficient conditions for $\lambda, \mu, f$ and $g$ such that the system,

$\left\{\begin{array}{l}u^{(n)}(t)+\lambda c(t) f(u(t), v(t))=0, \quad t \in(0, T), \quad n \in \mathbb{N}, \quad n \geq 2, \\ v^{(m)}(t)+\mu d(t) g(u(t), v(t))=0, \quad t \in(0, T), \quad m \in \mathbb{N}, \quad m \geq 2,\end{array}\right.$

with the boundary conditions $(B C)$, has positive solutions $(u(t) \geq 0, v(t) \geq 0$ for all $t \in[0, T]$ and $\|u\|+\|v\|>0)$. The system $\left(S_{1}\right)$ with $n=m=2$ and the multi-point boundary conditions

$$
\left\{\begin{array}{l}
\alpha u(0)-\beta u^{\prime}(0)=0, \quad u(T)=\sum_{i=1}^{p-2} a_{i} u\left(\xi_{i}\right), \quad p \in \mathbb{N}, \quad p \geq 3, \\
\gamma v(0)-\delta v^{\prime}(0)=0, \quad v(T)=\sum_{i=1}^{q-2} b_{i} v\left(\eta_{i}\right), \quad q \in \mathbb{N}, \quad q \geq 3,
\end{array}\right.
$$

has been investigated in [4]. Some particular cases of the above problems have been studied in $[6-9,12,13,15]$.

In recent years, the multi-point boundary value problems for secondorder or higher-order differential or difference equations/systems have been investigated by many authors, by using different methods such as fixed point theorems in cones, the Leray-Schauder continuation theorem and its nonlinear alternatives and the coincidence degree theory.

In Sect. 2, we shall present some auxiliary results which investigate two boundary value problems for higher-order equations (the problems (1)-(2) and (10)-(11) below). In Sect. 3, inspired by the paper [16], we shall prove some existence and multiplicity results for positive solutions with respect to a cone for our problem $(S)-(B C)$, which are based on three fixed point index theorems. Finally, in Sect. 4, we shall present some examples which illustrate our main results.

\section{Auxiliary results}

\subsection{Fixed point index theorems}

First, we shall recall some theorems concerning the index fixed point theory. Let $E$ be a real Banach space, $P \subset E$ a cone, " $\leq$ " the partial ordering defined by $P$ and $\theta$ the zero element in $E$. For $\varrho>0$, let $B_{\varrho}=\{u \in E,\|u\|<\varrho\}$. The proofs of our results are based on the following fixed point index theorems. 
Theorem 2.1. [1] Let $A: \bar{B}_{\varrho} \cap P \rightarrow P$ be a completely continuous operator which has no fixed point on $\partial B_{\varrho} \cap P$. If $\|A u\| \leq\|u\|$ for all $u \in \partial B_{\varrho} \cap P$, then $i\left(A, B_{\varrho} \cap P, P\right)=1$.

Theorem 2.2. [1] Let $A: \bar{B}_{\varrho} \cap P \rightarrow P$ be a completely continuous operator. If there exists $u_{0} \in P \backslash\{\theta\}$ such that $u-A u \neq \lambda u_{0}$, for all $\lambda \geq 0$ and $u \in \partial B_{\varrho} \cap P$, then $i\left(A, B_{\varrho} \cap P, P\right)=0$.

Theorem 2.3. [16] Let $A: \bar{B}_{\varrho} \cap P \rightarrow P$ be a completely continuous operator which has no fixed point on $\partial B_{\varrho} \cap P$. If there exists a linear operator $L: P \rightarrow P$ and $u_{0} \in P \backslash\{\theta\}$ such that

$$
\text { (i) } u_{0} \leq L u_{0}, \quad \text { (ii) } L u \leq A u, \quad \forall u \in \partial B_{\varrho} \cap P,
$$

then $i\left(A, B_{\varrho} \cap P, P\right)=0$.

\subsection{Higher-order differential equations with multi-point boundary conditions}

In this subsection, we shall present some auxiliary results (for the proofs see, for example, $[11,12])$ related to the $n$ th-order differential equation with $p$-point boundary conditions

$$
\begin{gathered}
u^{(n)}(t)+y(t)=0, \quad t \in(0, T), \\
u(0)=u^{\prime}(0)=\cdots=u^{(n-2)}(0)=0, \quad u(T)=\sum_{i=1}^{p-2} a_{i} u\left(\xi_{i}\right) .
\end{gathered}
$$

Lemma 2.1. If $d=T^{n-1}-\sum_{i=1}^{p-2} a_{i} \xi_{i}^{n-1} \neq 0,0<\xi_{1}<\cdots<\xi_{p-2}<T$ and $y \in C([0, T])$, then the solution of $(1)-(2)$ is given by

$$
\begin{aligned}
u(t)= & \frac{t^{n-1}}{d(n-1) !} \int_{0}^{T}(T-s)^{n-1} y(s) d s-\frac{t^{n-1}}{d(n-1) !} \sum_{i=1}^{p-2} a_{i} \int_{0}^{\xi_{i}}\left(\xi_{i}-s\right)^{n-1} y(s) d s \\
& -\frac{1}{(n-1) !} \int_{0}^{t}(t-s)^{n-1} y(s) d s, \quad 0 \leq t \leq T .
\end{aligned}
$$

Lemma 2.2. Under the assumptions of Lemma 2.1, the Green's function for the boundary value problem (1)-(2) is given by

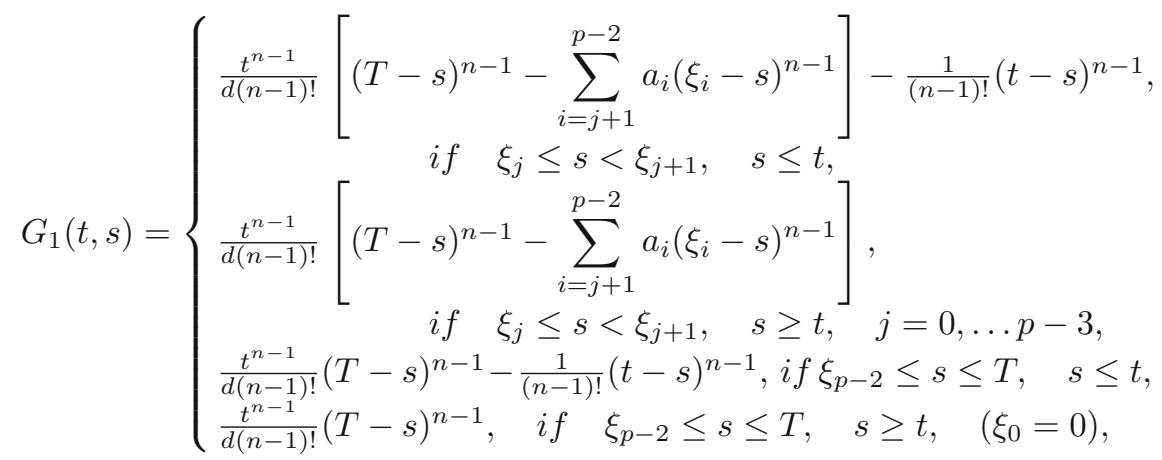

for all $(t, s) \in[0, T] \times[0, T]$. 
Using the Heaviside function on $\mathbb{R}, H(x)=1$ for $x \geq 0$, and $H(x)=0$ for $x<0$, the above Green's function can be written in a compact form

$$
\begin{aligned}
G_{1}(t, s)= & \frac{t^{n-1}}{d(n-1) !}\left[(T-s)^{n-1}-\sum_{i=1}^{p-2} a_{i}\left(\xi_{i}-s\right)^{n-1} H\left(\xi_{i}-s\right)\right] \\
& -\frac{1}{(n-1) !}(t-s)^{n-1} H(t-s), \quad(t, s) \in[0, T] \times[0, T] .
\end{aligned}
$$

By using the above Green's function the solution of problem (1)-(2) is expressed as $u(t)=\int_{0}^{T} G_{1}(t, s) y(s) d s$.

Lemma 2.3. Under the assumptions of Lemma 2.1, the Green's function for the boundary value problem (1)-(2) can be expressed as

$$
G_{1}(t, s)=g_{1}(t, s)+\frac{t^{n-1}}{d} \sum_{i=1}^{p-2} a_{i} g_{1}\left(\xi_{i}, s\right),
$$

where

$g_{1}(t, s)=\frac{1}{(n-1) ! T^{n-1}}\left\{\begin{array}{l}t^{n-1}(T-s)^{n-1}-T^{n-1}(t-s)^{n-1}, \quad 0 \leq s \leq t \leq T, \\ t^{n-1}(T-s)^{n-1}, \quad 0 \leq t \leq s \leq T .\end{array}\right.$

Proof. If $s \leq t$ and $\xi_{j} \leq s<\xi_{j+1}$ for $j \in\{0,1, \ldots, p-3\}$, we have

$$
\begin{aligned}
G_{1}(t, s)= & \frac{1}{d(n-1) !}\left[t^{n-1}(T-s)^{n-1}-t^{n-1} \sum_{i=j+1}^{p-2} a_{i}\left(\xi_{i}-s\right)^{n-1}\right. \\
& \left.-(t-s)^{n-1} T^{n-1}+(t-s)^{n-1} \sum_{i=1}^{p-2} a_{i} \xi_{i}^{n-1}\right] \\
= & \frac{1}{d(n-1) !}\left[t^{n-1}(T-s)^{n-1}-T^{n-1}(t-s)^{n-1}+(t-s)^{n-1} \sum_{i=1}^{p-2} a_{i} \xi_{i}^{n-1}\right. \\
& -\frac{t^{n-1}(T-s)^{n-1}}{T^{n-1}} \sum_{i=1}^{p-2} a_{i} \xi_{i}^{n-1}+\frac{t^{n-1}(T-s)^{n-1}}{T^{n-1}} \sum_{i=1}^{j} a_{i} \xi_{i}^{n-1} \\
& \left.+\frac{t^{n-1}(T-s)^{n-1}}{T^{n-1}} \sum_{i=j+1}^{p-2} a_{i} \xi_{i}^{n-1}-t^{n-1} \sum_{i=j+1}^{p-2} a_{i}\left(\xi_{i}-s\right)^{n-1}\right] \\
= & \frac{1}{d(n-1) ! T^{n-1}}\left\{\left(T^{n-1}-\sum_{i=1}^{p-2} a_{i} \xi_{i}^{n-1}\right)\left[t^{n-1}(T-s)^{n-1}-T^{n-1}(t-s)^{n-1}\right]\right. \\
& +t^{n-1}(T-s)^{n-1} \sum_{i=1}^{j} a_{i} \xi_{i}^{n-1}+t^{n-1} \sum_{i=j+1}^{p-2} a_{i}\left[\xi_{i}^{n-1}(T-s)^{n-1}\right. \\
& -\frac{1}{\left.\left.(n-1) ! T^{n-1}\left(\xi_{i}-s\right)^{n-1}\right]\right\}} \\
= & \left.\frac{1}{n-1}(T-s)^{n-1}-T^{n-1}(t-s)^{n-1}\right]
\end{aligned}
$$




$$
\begin{aligned}
& +\frac{t^{n-1}}{d(n-1) ! T^{n-1}} \sum_{i=1}^{j} a_{i} \xi_{i}^{n-1}(T-s)^{n-1} \\
& +\frac{t^{n-1}}{d(n-1) ! T^{n-1}} \sum_{i=j+1}^{p-2} a_{i}\left[\xi_{i}^{n-1}(T-s)^{n-1}-T^{n-1}\left(\xi_{i}-s\right)^{n-1}\right] \\
& =g_{1}(t, s)+\frac{t^{n-1}}{d} \sum_{i=1}^{p-2} a_{i} g_{1}\left(\xi_{i}, s\right) .
\end{aligned}
$$

We use the convention that $\sum_{i=1}^{0} \alpha_{i}=0$.

If $s \geq t, \xi_{j} \leq s<\xi_{j+1}$ for $j \in\{0,1, \ldots, p-3\}$, we obtain

$$
\begin{aligned}
& G_{1}(t, s)=\frac{1}{d(n-1) !}\left[t^{n-1}(T-s)^{n-1}-t^{n-1} \sum_{i=j+1}^{p-2} a_{i}\left(\xi_{i}-s\right)^{n-1}\right] \\
& =\frac{1}{d(n-1) !}\left[t^{n-1}(T-s)^{n-1}-\frac{t^{n-1}(T-s)^{n-1}}{T^{n-1}} \sum_{i=1}^{p-2} a_{i} \xi_{i}^{n-1}\right. \\
& +\frac{t^{n-1}(T-s)^{n-1}}{T^{n-1}} \sum_{i=1}^{j} a_{i} \xi_{i}^{n-1}+\frac{t^{n-1}(T-s)^{n-1}}{T^{n-1}} \\
& \left.\times \sum_{i=j+1}^{p-2} a_{i} \xi_{i}^{n-1}-t^{n-1} \sum_{i=j+1}^{p-2} a_{i}\left(\xi_{i}-s\right)^{n-1}\right]=\frac{t^{n-1}(T-s)^{n-1}}{d(n-1) ! T^{n-1}} \\
& \times\left(T^{n-1}-\sum_{i=1}^{p-2} a_{i} \xi_{i}^{n-1}\right)+\frac{t^{n-1}}{d(n-1) ! T^{n-1}} \sum_{i=1}^{j} a_{i} \xi_{i}^{n-1}(T-s)^{n-1} \\
& +\frac{t^{n-1}}{d(n-1) ! T^{n-1}} \sum_{i=j+1}^{p-2} a_{i}\left[\xi_{i}^{n-1}(T-s)^{n-1}-T^{n-1}\left(\xi_{i}-s\right)^{n-1}\right] \\
& =\frac{1}{(n-1) ! T^{n-1}} t^{n-1}(T-s)^{n-1}+\frac{t^{n-1}}{d(n-1) !} \sum_{i=1}^{j} a_{i} \xi_{i}^{n-1}(T-s)^{n-1} \\
& +\frac{t^{n-1}}{d(n-1) ! T^{n-1}} \sum_{i=j+1}^{p-2} a_{i}\left[\xi_{i}^{n-1}(T-s)^{n-1}-T^{n-1}\left(\xi_{i}-s\right)^{n-1}\right] \\
& =g_{1}(t, s)+\frac{t^{n-1}}{d} \sum_{i=1}^{p-2} a_{i} g_{1}\left(\xi_{i}, s\right)
\end{aligned}
$$

If $s \leq t$ and $\xi_{p-2} \leq s \leq T$, we have

$$
\begin{aligned}
G_{1}(t, s) & =\frac{t^{n-1}}{d(n-1) !}(T-s)^{n-1}-\frac{1}{(n-1) !}(t-s)^{n-1} \\
& =\frac{t^{n-1}}{d(n-1) !}(T-s)^{n-1}-\frac{1}{d(n-1) ! T^{n-1}} t^{n-1}(T-s)^{n-1} \sum_{i=1}^{p-2} a_{i} \xi_{i}^{n-1}
\end{aligned}
$$




$$
\begin{aligned}
& -\frac{1}{(n-1) !}(t-s)^{n-1}+\frac{t^{n-1}}{d(n-1) ! T^{n-1}} \sum_{i=1}^{p-2} a_{i} \xi_{i}^{n-1}(T-s)^{n-1} \\
= & \left(T^{n-1}-\sum_{i=1}^{p-2} a_{i} \xi_{i}^{n-1}\right) \frac{1}{d(n-1) ! T^{n-1}} t^{n-1}(T-s)^{n-1} \\
& -\frac{1}{(n-1) !}(t-s)^{n-1}+\frac{t^{n-1}}{d(n-1) ! T^{n-1}} \sum_{i=1}^{p-2} a_{i} \xi_{i}^{n-1}(T-s)^{n-1} \\
= & \frac{1}{(n-1) ! T^{n-1}}\left[t^{n-1}(T-s)^{n-1}-T^{n-1}(t-s)^{n-1}\right] \\
& +\frac{t^{n-1}}{d(n-1) ! T^{n-1}} \sum_{i=1}^{p-2} a_{i} \xi_{i}^{n-1}(T-s)^{n-1} \\
= & g_{1}(t, s)+\frac{t^{n-1}}{d} \sum_{i=1}^{p-2} a_{i} g_{1}\left(\xi_{i}, s\right) .
\end{aligned}
$$

If $s \geq t$ and $\xi_{p-2} \leq s \leq T$, we obtain

$$
\begin{aligned}
G_{1}(t, s)= & \frac{t^{n-1}(T-s)^{n-1}}{d(n-1) !}=\frac{t^{n-1}(T-s)^{n-1}}{d(n-1) !}-\frac{t^{n-1}(T-s)^{n-1}}{d(n-1) ! T^{n-1}} \sum_{i=1}^{p-2} a_{i} \xi_{i}^{n-1} \\
& +\frac{t^{n-1}(T-s)^{n-1}}{d(n-1) ! T^{n-1}} \sum_{i=1}^{p-2} a_{i} \xi_{i}^{n-1} \\
= & \frac{t^{n-1}(T-s)^{n-1}}{d(n-1) ! T^{n-1}}\left(T^{n-1}-\sum_{i=1}^{p-2} a_{i} \xi_{i}^{n-1}\right) \\
& +\frac{t^{n-1}}{d(n-1) ! T^{n-1}} \sum_{i=1}^{p-2} a_{i} \xi_{i}^{n-1}(T-s)^{n-1}=\frac{1}{(n-1) ! T^{n-1}} t^{n-1}(T-s)^{n-1} \\
& +\frac{t^{n-1}}{d(n-1) ! T^{n-1}} \sum_{i=1}^{p-2} a_{i} \xi_{i}^{n-1}(T-s)^{n-1} \\
= & g_{1}(t, s)+\frac{t^{n-1}}{d} \sum_{i=1}^{p-2} a_{i} g_{1}\left(\xi_{i}, s\right) .
\end{aligned}
$$

Therefore, in every case from above, we obtain the relation (3).

If $a_{i}=0$ for $i=1, \ldots, p-2$, then $G_{1}(t, s)=g_{1}(t, s)$ is the Green's function for the problem

$$
\begin{gathered}
w^{(n)}(t)+y_{0}(t)=0, \quad t \in(0, T), \\
w(0)=w^{\prime}(0)=\cdots=w^{(n-2)}(0)=0, \quad w(T)=0 .
\end{gathered}
$$


The solution of the above problem is

$$
\begin{aligned}
w(t)= & \frac{t^{n-1}}{(n-1) ! T^{n-1}} \int_{0}^{T}(T-s)^{n-1} y_{0}(s) d s \\
& -\frac{1}{(n-1) !} \int_{0}^{t}(t-s)^{n-1} y_{0}(s) d s, \quad t \in[0, T] \\
\Leftrightarrow \quad w(t)= & \int_{0}^{T} g_{1}(t, s) y_{0}(s) d s, \quad t \in[0, T],
\end{aligned}
$$

where $g_{1}$ is given in (4).

Therefore, the function $g_{1}$ satisfies the conditions $g_{1}(0, s)=0, \frac{\partial g_{1}}{\partial t}(0, s)=$ $0, \ldots \frac{\partial^{n-2} g_{1}}{\partial t^{n-2}}(0, s)=0$ for all $s \in[0, T]$. Moreover, we have $g_{1}(t, 0)=0$ and $g_{1}(t, T)=0$ for all $t \in[0, T]$. We also observe that $g_{1}$ is a continuous function on $[0, T] \times[0, T]$ and $g_{1}(t, s) \geq 0$ for all $(t, s) \in[0, T] \times[0, T]$. Indeed, if $0 \leq t \leq s \leq T$, it is obvious that $g_{1}(t, s) \geq 0$. For $0 \leq s \leq t \leq T$, we have

$$
g_{1}(t, s)=\frac{1}{(n-1) ! T^{n-1}}\left[(t T-t s)^{n-1}-(t T-s T)^{n-1}\right] \geq 0,
$$

because $t T-t s \geq t T-s T \Leftrightarrow t \leq T$.

By using the above properties of $g_{1}$ and similar arguments as those used in the proof of Lemma 3.3 from [10], we obtain the following result.

Lemma 2.4. The Green's function $g_{1}$ of problem (5)-(6) satisfies the inequalities

(a) $g_{1}(t, s) \leq g_{1}\left(\theta_{1}(s), s\right)$, for all $(t, s) \in[0, T] \times[0, T]$,

(b) For any $c \in\left(0, \frac{T}{2}\right)$,

$$
\min _{t \in[c, T-c]} g_{1}(t, s) \geq \frac{c^{n-1}}{T^{n-1}} g_{1}\left(\theta_{1}(s), s\right), \quad \text { for all } s \in[0, T]
$$

where $\theta_{1}(s)=s$ if $n=2$ and $\theta_{1}(s)= \begin{cases}\frac{s}{1-\left(1-\frac{s}{T}\right)^{\frac{n-1}{n-2}},}, \quad s \in(0, T], & \\ \frac{T(n-2)}{n-1}, s=0, & \text { if } n \geq 3 .\end{cases}$

In the case $n \geq 3$, we choose the values of $\theta_{1}$ in $s=0$ and $s=T$ such that $\theta_{1}$ be a continuous function on $[0, T]$.

Lemma 2.5. Assume that $d>0,0<\xi_{1}<\cdots<\xi_{p-2}<T, a_{i} \geq 0$ for all $i=1, \ldots, p-2$. Then the Green's function $G_{1}$ of the problem (1)-(2) is continuous on $[0, T] \times[0, T]$ and satisfies $G_{1}(t, s) \geq 0$ for all $(t, s) \in[0, T] \times[0, T]$. Moreover, if $y \in C([0, T])$ satisfies $y(t) \geq 0$ for all $t \in[0, T]$, then the unique solution $u$ of problem (1)-(2) satisfies $u(t) \geq 0$ for all $t \in[0, T]$.

The proof of Lemma 2.5 is immediate by using the nonnegativity property of $g_{1}$ and the expressions for $G_{1}$ and $u$.

Lemma 2.6. Assume that $d>0,0<\xi_{1}<\cdots<\xi_{p-2}<T, a_{i} \geq 0$ for all $i=1, \ldots, p-2$. Then the Green's function $G_{1}$ of problem (1)-(2) satisfies the 
inequalities

(a) $G_{1}(t, s) \leq I_{1}(s), \quad \forall(t, s) \in[0, T] \times[0, T]$,

where $I_{1}(s)=g_{1}\left(\theta_{1}(s), s\right)+\frac{T^{n-1}}{d} \sum_{i=1}^{p-2} a_{i} g_{1}\left(\xi_{i}, s\right), \quad \forall s \in[0, T]$;

(b) For every $c \in\left(0, \frac{T}{2}\right)$,

$$
\min _{t \in[c, T-c]} G_{1}(t, s) \geq \frac{c^{n-1}}{T^{n-1}} I_{1}(s), \quad \forall s \in[0, T] .
$$

Proof. From (3) and Lemma 2.4 we obtain the inequality (a), and for any $c \in\left(0, \frac{T}{2}\right)$, we have

$$
\begin{aligned}
G_{1}(t, s) & \geq \frac{c^{n-1}}{T^{n-1}} g_{1}\left(\theta_{1}(s), s\right)+\frac{c^{n-1}}{d} \sum_{i=1}^{p-2} a_{i} g_{1}\left(\xi_{i}, s\right) \\
& =\frac{c^{n-1}}{T^{n-1}}\left[g_{1}\left(\theta_{1}(s), s\right)+\frac{T^{n-1}}{d} \sum_{i=1}^{p-2} a_{i} g_{1}\left(\xi_{i}, s\right)\right] \\
& =\frac{c^{n-1}}{T^{n-1}} I_{1}(s), \quad \forall s \in[0, T], \quad t \in[c, T-c],
\end{aligned}
$$

and then $\min _{t \in[c, T-c]} G_{1}(t, s) \geq \frac{c^{n-1}}{T^{n-1}} I_{1}(s)$ for all $s \in[0, T]$.

Lemma 2.7. Assume that $d>0,0<\xi_{1}<\cdots<\xi_{p-2}<T, a_{i} \geq 0$ for all $i=1, \ldots, p-2, c \in\left(0, \frac{T}{2}\right)$ and $y \in C([0, T])$ satisfies $y(t) \geq 0$ for all $t \in[0, T]$. Then the solution $u(t), t \in[0, T]$ of problem (1)-(2) satisfies the inequality

$$
\min _{t \in[c, T-c]} u(t) \geq \frac{c^{n-1}}{T^{n-1}} \max _{t^{\prime} \in[0, T]} u\left(t^{\prime}\right) .
$$

Proof. By using the inequalities (7) and (8), we obtain

$$
\begin{aligned}
u(t) & =\int_{0}^{T} G_{1}(t, s) y(s) d s \geq \frac{c^{n-1}}{T^{n-1}} \int_{0}^{T} I_{1}(s) y(s) d s \\
& \geq \frac{c^{n-1}}{T^{n-1}} \int_{0}^{T} G_{1}\left(t^{\prime}, s\right) y(s) d s=\frac{c^{n-1}}{T^{n-1}} u\left(t^{\prime}\right), \quad \forall t \in[c, T-c], \quad t^{\prime} \in[0, T] .
\end{aligned}
$$

Therefore, we conclude

$$
\min _{t \in[c, T-c]} u(t) \geq \frac{c^{n-1}}{T^{n-1}} \max _{t^{\prime} \in[0, T]} u\left(t^{\prime}\right),
$$

that is the inequality (9).

We can also formulate similar results as Lemmas 2.1-2.7 above for the boundary value problem

$$
\begin{gathered}
v^{(m)}(t)+h(t)=0, \quad t \in(0, T), \\
v(0)=v^{\prime}(0)=\cdots=v^{(m-2)}(0)=0, \quad v(T)=\sum_{i=1}^{q-2} b_{i} v\left(\eta_{i}\right),
\end{gathered}
$$


where $0<\eta_{1}<\cdots<\eta_{q-2}<T, b_{i} \geq 0$ for $i=1, \ldots, q-2$ and $h \in C([0, T])$. If $e=T^{m-1}-\sum_{i=1}^{q-2} b_{i} \eta_{i}^{m-1} \neq 0$, we denote by $G_{2}$ the Green's function associated to problem (10)-(11) and defined in a similar manner as $G_{1}$. We also denote by $g_{2}, \theta_{2}$ and $I_{2}$ the corresponding functions for (10)-(11) defined in a similar manner as $g_{1}, \theta_{1}$ and $I_{1}$, respectively.

\section{Main results}

In this section, we shall investigate the existence and multiplicity of positive solutions for our problem $(S)-(B C)$, under various assumptions on $f$ and $g$.

We present the assumptions that we shall use in the sequel

(H1) $0<\xi_{1}<\cdots<\xi_{p-2}<T, a_{i} \geq 0, i=1, \ldots, p-2, d=T^{n-1}-$ $\sum_{i=1}^{p-2} a_{i} \xi_{i}^{n-1}>0,0<\eta_{1}<\cdots<\eta_{q-2}<T, b_{i} \geq 0, i=1, \ldots, q-2, e=$ $T^{m-1}-\sum_{i=1}^{q-2} b_{i} \eta_{i}^{m-1}>0$.

$(H 2)$ The functions $f, g \in C([0, T] \times[0, \infty),[0, \infty))$ and $f(t, 0)=0, g(t, 0)=0$ for all $t \in[0, T]$.

(H3) There exists a positive constant $p_{1} \in(0,1]$ such that

(i) $f_{\infty}^{i}=\liminf _{u \rightarrow \infty} \inf _{t \in[0, T]} \frac{f(t, u)}{u^{p_{1}}} \in(0, \infty] ; \quad$ (ii) $g_{\infty}^{i}=\liminf _{u \rightarrow \infty} \inf _{t \in[0, T]} \frac{g(t, u)}{u^{1 / p_{1}}}=\infty$.

(H4) There exists a positive constant $q_{1} \in(0, \infty)$ such that

(i) $f_{0}^{s}=\limsup _{u \rightarrow 0^{+}} \sup _{t \in[0, T]} \frac{f(t, u)}{u^{q_{1}}} \in[0, \infty) ; \quad$ (ii) $g_{0}^{s}=\limsup _{u \rightarrow 0^{+}} \sup _{t \in[0, T]} \frac{g(t, u)}{u^{1 / q_{1}}}=0$.

(H5) There exists a positive constant $r \in(0, \infty)$ such that

(i) $f_{\infty}^{s}=\limsup _{u \rightarrow \infty} \sup _{t \in[0, T]} \frac{f(t, u)}{u^{r}} \in[0, \infty) ; \quad g_{\infty}^{s}=\limsup _{u \rightarrow \infty} \sup _{t \in[0, T]} \frac{g(t, u)}{u^{1 / r}}=0$.

(H6) The following conditions are satisfied

$$
f_{0}^{i}=\liminf _{u \rightarrow 0^{+}} \inf _{t \in[0, T]} \frac{f(t, u)}{u} \in(0, \infty] ; \quad g_{0}^{i}=\liminf _{u \rightarrow 0^{+}} \inf _{t \in[0, T]} \frac{g(t, u)}{u}=\infty .
$$

(H7) For each $t \in[0, T], f(t, u)$ and $g(t, u)$ are nondecreasing with respect to $u$, and there exists a constant $N>0$ such that

$$
f\left(t, m_{0} \int_{0}^{T} g(s, N) d s\right)<\frac{N}{m_{0}}, \quad \forall t \in[0, T]
$$

where $m_{0}=\max \left\{K_{1} T, K_{2}\right\}, K_{1}=\max _{s \in[0, T]} I_{1}(s), K_{2}=\max _{s \in[0, T]} I_{2}(s)$ and $I_{1}, I_{2}$ are defined in Sect. 2 .

The pair of functions $(u, v) \in C^{n}([0, T]) \times C^{m}([0, T])$ is a solution for our problem $(S)-(B C)$ if and only if $(u, v) \in C([0, T]) \times C([0, T])$ is a solution for the nonlinear integral system

$$
\begin{cases}u(t)=\int_{0}^{T} G_{1}(t, s) f(s, v(s)) d s, & t \in[0, T], \\ v(t)=\int_{0}^{T} G_{2}(t, s) g(s, u(s)) d s, & t \in[0, T] .\end{cases}
$$


Besides, the system (12) can be written as the nonlinear integral system

$$
\left\{\begin{array}{l}
u(t)=\int_{0}^{T} G_{1}(t, s) f\left(s, \int_{0}^{T} G_{2}(s, \tau) g(\tau, u(\tau)) d \tau\right) d s, \quad t \in[0, T], \\
v(t)=\int_{0}^{T} G_{2}(t, s) g(s, u(s)) d s, \quad t \in[0, T] .
\end{array}\right.
$$

We consider the Banach space $X=C([0, T])$ with supremum norm $\|\cdot\|$ and define the cone $P \subset X$ by $P=\{u \in X, u(t) \geq 0, \forall t \in[0, T]\}$.

We also define the operators $A: P \rightarrow X$ by

$$
(A u)(t)=\int_{0}^{T} G_{1}(t, s) f\left(s, \int_{0}^{T} G_{2}(s, \tau) g(\tau, u(\tau)) d \tau\right) d s, \quad t \in[0, T],
$$

and $B: P \rightarrow X, C: P \rightarrow X$ by

$$
(B u)(t)=\int_{0}^{T} G_{1}(t, s) u(s) d s, \quad(C u)(t)=\int_{0}^{T} G_{2}(t, s) u(s) d s, \quad t \in[0, T] .
$$

Under the assumptions $(H 1)$ and $(H 2)$, using also Lemma 2.5, it is easy to see that $A, B$ and $C$ are completely continuous from $P$ to $P$. Thus the existence and multiplicity of positive solutions of the system $(S)-(B C)$ are equivalent to the existence and multiplicity of fixed points of the operator $A$.

Theorem 3.1. Assume that $(H 1)-(H 4)$ hold. Then the problem $(S)-(B C)$ has at least one positive solution $(u(t), v(t)), t \in[0, T]$.

Proof. From assumption (i) of (H3), we conclude that there exist $C_{1}, C_{2}>0$ such that

$$
f(t, u) \geq C_{1} u^{p_{1}}-C_{2}, \quad \forall(t, u) \in[0, T] \times[0, \infty) .
$$

Then for $u \in P$, by using (13), the reverse form of Holder's inequality and Lemma 2.6, we have for $p_{1} \in(0,1)$

$$
\begin{aligned}
(A u)(t)= & \int_{0}^{T} G_{1}(t, s) f\left(s, \int_{0}^{T} G_{2}(s, \tau) g(\tau, u(\tau)) d \tau\right) d s \\
\geq & \int_{0}^{T} G_{1}(t, s)\left[C_{1}\left(\int_{0}^{T} G_{2}(s, \tau) g(\tau, u(\tau)) d \tau\right)^{p_{1}}-C_{2}\right] d s \\
\geq & \int_{0}^{T} G_{1}(t, s)\left[C_{1} \int_{0}^{T}\left(G_{2}(s, \tau) g(\tau, u(\tau))\right)^{p_{1}} d \tau\left(\int_{0}^{T} d \tau\right)^{p_{1} / q_{0}}\right] d s \\
& -C_{2} \int_{0}^{T} I_{1}(s) d s=C_{1} T^{p_{1} / q_{0}} \int_{0}^{T} G_{1}(t, s) \\
& \times\left(\int_{0}^{T}\left(G_{2}(s, \tau)\right)^{p_{1}}(g(\tau, u(\tau)))^{p_{1}} d \tau\right) d s-C_{3}, \quad \forall t \in[0, T],
\end{aligned}
$$

where $q_{0}=p_{1} /\left(p_{1}-1\right)$ and $C_{3}=C_{2} \int_{0}^{T} I_{1}(s) d s$. 
Therefore, for $u \in P$ and $p_{1} \in(0,1]$, we have

$$
\begin{gathered}
(A u)(t) \geq \widetilde{C}_{1} \int_{0}^{T} G_{1}(t, s)\left(\int_{0}^{T}\left(G_{2}(s, \tau)\right)^{p_{1}}(g(\tau, u(\tau)))^{p_{1}} d \tau\right) d s-C_{3}, \\
\forall t \in[0, T]
\end{gathered}
$$

where $\widetilde{C}_{1}=C_{1} T^{p_{1} / q_{0}}$ for $p_{1} \in(0,1)$ and $\widetilde{C}_{1}=C_{1}$ for $p_{1}=1$.

For $c \in\left(0, \frac{T}{2}\right)$, we define the cone

$$
P_{0}=\left\{u \in P ; \inf _{t \in[c, T-c]} u(t) \geq \gamma\|u\|\right\},
$$

where $\gamma=\min \left\{c^{n-1} / T^{n-1}, c^{m-1} / T^{m-1}\right\}$.

From our assumptions and Lemma 2.7, it can be shown that for any $y \in P$ the functions $u(t)=(B y)(t)$ and $v(t)=(C y)(t)$ satisfy the inequalities

$$
\inf _{t \in[c, T-c]} u(t) \geq \frac{c^{n-1}}{T^{n-1}}\|u\| \geq \gamma\|u\|, \quad \inf _{t \in[c, T-c]} v(t) \geq \frac{c^{m-1}}{T^{m-1}}\|v\| \geq \gamma\|v\| .
$$

So, $u=B y \in P_{0}, v=C y \in P_{0}$. Therefore, we deduce that $B(P) \subset P_{0}, C(P) \subset$ $P_{0}$.

Now we consider the function $u_{0}(t), t \in[0, T]$, the solution of problem (1)(2) with $y=y_{0}$, where $y_{0}(t)=1$ for all $t \in[0, T]$. Then $u_{0}(t)=\int_{0}^{T} G_{1}(t, s) d s=$ $\left(B y_{0}\right)(t), t \in[0, T]$. Obviously, we have $u_{0}(t) \geq 0$ for all $t \in[0, T]$. We also consider the set

$$
M=\left\{u \in P \text {; there exists } \lambda \geq 0 \text { such that } u=A u+\lambda u_{0}\right\} .
$$

We will show that $M \subset P_{0}$ and $M$ is a bounded subset of $X$. If $u \in M$, then there exists $\lambda \geq 0$ such that $u(t)=(A u)(t)+\lambda u_{0}(t), t \in[0, T]$. From definition of $u_{0}$, we have

$u(t)=(A u)(t)+\lambda\left(B y_{0}\right)(t)=B(F u(t))+\lambda\left(B y_{0}\right)(t)=B\left(F u(t)+\lambda y_{0}(t)\right) \in P_{0}$,

where $F: P \rightarrow P$ is defined by $(F u)(t)=f\left(t, \int_{0}^{T} G_{2}(t, s) g(s, u(s)) d s\right)$. Therefore, $M \subset P_{0}$, and from the definition of $P_{0}$, we have

$$
\|u\| \leq \frac{1}{\gamma} \inf _{t \in[c, T-c]} u(t), \quad \forall u \in M
$$

From (ii) of assumption $(H 3)$, we conclude that for $\varepsilon_{0}=\left(\frac{2 T^{p_{1}(m-1)+n-1}}{\widetilde{C}_{1} m_{1} m_{2} c^{p_{1}(m-1)+n-1}}\right)^{1 / p_{1}}$ $>0$ there exists $C_{4}>0$ such that

$$
(g(t, u))^{p_{1}} \geq \varepsilon_{0}^{p_{1}} u-C_{4}, \quad \forall(t, u) \in[0, T] \times[0, \infty),
$$

where $m_{1}=\int_{c}^{T-c} I_{1}(\tau) d \tau>0, m_{2}=\int_{c}^{T-c}\left(I_{2}(\tau)\right)^{p_{1}} d \tau>0$. 
For $u \in M$ and $t \in[c, T-c]$, by using Lemma 2.6 and the relations (14), (16), it follows that

$$
\begin{aligned}
u(t) & =(A u)(t)+\lambda u_{0}(t) \geq(A u)(t) \\
& \geq \widetilde{C}_{1} \int_{0}^{T} G_{1}(t, s)\left[\int_{0}^{T}\left(G_{2}(s, \tau)\right)^{p_{1}}(g(\tau, u(\tau)))^{p_{1}} d \tau\right] d s-C_{3} \\
& \geq \widetilde{C}_{1} \int_{c}^{T-c} G_{1}(t, s)\left[\int_{c}^{T-c}\left(G_{2}(s, \tau)\right)^{p_{1}}(g(\tau, u(\tau)))^{p_{1}} d \tau\right] d s-C_{3} \\
& \geq \frac{\widetilde{C}_{1} c^{p_{1}(m-1)+n-1}}{T^{p_{1}(m-1)+n-1}} \int_{c}^{T-c} I_{1}(s)\left(\int_{c}^{T-c}\left(I_{2}(\tau)\right)^{p_{1}}(g(\tau, u(\tau)))^{p_{1}} d \tau\right) d s-C_{3} \\
& \geq \frac{\widetilde{C}_{1} c^{p_{1}(m-1)+n-1}}{T^{p_{1}(m-1)+n-1}}\left(\int_{c}^{T-c} I_{1}(s) d s\right)\left(\int_{c}^{T-c}\left(I_{2}(\tau)\right)^{p_{1}}\left(\varepsilon_{0}^{p_{1}} u(\tau)-C_{4}\right) d \tau\right)-C_{3} \\
& =\frac{\widetilde{C}_{1} \varepsilon_{0}^{p_{1}} c^{p_{1}(m-1)+n-1}}{T^{p_{1}(m-1)+n-1}}\left(\int_{c}^{T-c} I_{1}(s) d s\right)\left(\int_{c}^{T-c}\left(I_{2}(\tau)\right)^{p_{1}} u(\tau) d \tau\right)-C_{5} \\
& \geq \frac{\widetilde{C}_{1} \varepsilon_{0}^{p_{1}} c^{p_{1}(m-1)+n-1}}{T^{p_{1}(m-1)+n-1}}\left(\int_{c}^{T-c} I_{1}(s) d s\right)\left(\int_{c}^{T-c}\left(I_{2}(\tau)\right)^{p_{1}} d \tau\right) \inf _{\tau \in[c, T-c]} u(\tau)-C_{5} \\
& =2 \inf _{\tau \in[c, T-c]} u(\tau)-C_{5},
\end{aligned}
$$

where $C_{5}=C_{3}+\frac{C_{4} \widetilde{C}_{1} m_{1} m_{2} c^{p_{1}(m-1)+n-1}}{T^{p_{1}(m-1)+n-1}}>0$.

Hence, $\inf _{t \in[c, T-c]} u(t) \geq 2 \inf _{t \in[c, T-c]} u(t)-C_{5}$, and so

$$
\inf _{t \in[c, T-c]} u(t) \leq C_{5}, \quad \forall u \in M .
$$

Now from relations (15) and (17), it can be shown that $\|u\| \leq$ $\frac{1}{\gamma} \inf _{t \in[c, T-c]} u(t) \leq \frac{C_{5}}{\gamma}$, for all $u \in M$, that is, $M$ is a bounded subset of $X$.

Besides, there exists a sufficiently large $L>0$ such that

$$
u \neq A u+\lambda u_{0}, \quad \forall u \in \partial B_{L} \cap P, \quad \forall \lambda \geq 0 .
$$

From Theorem 2.2, we deduce that

$$
i\left(A, B_{L} \cap P, P\right)=0 .
$$

Next, from (i) of assumption (H4), we conclude that there exists $M_{0}>0$ such that

$$
f(t, u) \leq M_{0} u^{q}, \quad \forall(t, u) \in[0, T] \times[0,1] .
$$

From (ii) of assumption $(H 4)$ and $(H 2)$, it can be shown that for $\varepsilon_{1}=$ $\min \left\{\frac{1}{M_{2}},\left(\frac{1}{2 M_{0} M_{1} M_{2}^{q_{1}}}\right)^{1 / q_{1}}\right\}>0$, there exists $\delta_{1} \in(0,1)$ such that $g(t, u) \leq$ $\varepsilon_{1} u^{1 / q_{1}}$ for all $(t, u) \in[0, T] \times\left[0, \delta_{1}\right]$, where $M_{1}=\int_{0}^{T} I_{1}(s) d s>0, M_{2}=$ $\int_{0}^{T} I_{2}(s) d s>0$. Hence, we obtain

$$
\begin{aligned}
\int_{0}^{T} G_{2}(t, s) g(s, u(s)) d s & \leq \varepsilon_{1} \int_{0}^{T} I_{2}(s)(u(s))^{1 / q_{1}} d s \\
& \leq \varepsilon_{1} M_{2}\|u\|^{1 / q_{1}} \leq 1, \quad \forall u \in \bar{B}_{\delta_{1}} \cap P, \quad \forall t \in[0, T] .
\end{aligned}
$$


Therefore, by (19) and (20) we deduce

$$
\begin{aligned}
(A u)(t)= & \int_{0}^{T} G_{1}(t, s) f\left(s, \int_{0}^{T} G_{2}(s, \tau) g(\tau, u(\tau)) d \tau\right) d s \\
\leq & M_{0} \int_{0}^{T} G_{1}(t, s)\left(\int_{0}^{T} G_{2}(s, \tau) g(\tau, u(\tau)) d \tau\right)^{q_{1}} d s \\
\leq & M_{0} \varepsilon_{1}^{q_{1}} M_{2}^{q_{1}}\|u\| \int_{0}^{T} I_{1}(s) d s=M_{0} \varepsilon_{1}^{q_{1}} M_{1} M_{2}^{q_{1}}\|u\| \leq \frac{1}{2}\|u\|, \\
& \forall u \in \bar{B}_{\delta_{1}} \cap P, \quad t \in[0, T] .
\end{aligned}
$$

This implies that $\|A u\| \leq \frac{1}{2}\|u\|, \quad \forall u \in \partial B_{\delta_{1}} \cap P$. From Theorem 2.1, we conclude

$$
i\left(A, B_{\delta_{1}} \cap P, P\right)=1 \text {. }
$$

Combining (18) and (21), we obtain

$$
i\left(A,\left(B_{L} \backslash \bar{B}_{\delta_{1}}\right) \cap P, P\right)=i\left(A, B_{L} \cap P, P\right)-i\left(A, B_{\delta_{1}} \cap P, P\right)=-1 .
$$

We conclude that $A$ has at least one fixed point $u_{1} \in\left(B_{L} \backslash \bar{B}_{\delta_{1}}\right) \cap P$, that is $\delta_{1}<\left\|u_{1}\right\|<L$.

Let $v_{1}(t)=\int_{0}^{T} G_{2}(t, s) g\left(s, u_{1}(s)\right) d s$. Then $\left(u_{1}, v_{1}\right) \in P \times P$ is a solution of $(S)-(B C)$. In addition $\left\|v_{1}\right\|>0$. Indeed, if we suppose that $v_{1}(t)=0$, for all $t \in[0, T]$, then by using $(H 2)$ we have $f\left(s, v_{1}(s)\right)=f(s, 0)=0$, for all $s \in[0, T]$. This implies $u_{1}(t)=\int_{0}^{T} G_{1}(t, s) f\left(s, v_{1}(s)\right) d s=0$, for all $t \in[0, T]$, which contradicts $\left\|u_{1}\right\|>0$. By using Theorem 1.1 from [11] (see [2]), we obtain $u_{1}(t)>0$ and $v_{1}(t)>0$ for all $t \in(0, T-c]$. The proof of Theorem 3.1 is completed.

Theorem 3.2. Assume that $(H 1),(H 2),(H 5)$ and $(H 6)$ hold. Then the problem $(S)-(B C)$ has at least one positive solution $(u(t), v(t)), t \in[0, T]$.

Proof. From the assumption (H5)(i), we deduce that there exist $C_{6}, C_{7}>0$ such that

$$
f(t, u) \leq C_{6} u^{r}+C_{7}, \quad \forall(t, u) \in[0, T] \times[0, \infty) .
$$

From $(H 5)(\mathrm{ii})$, we conclude that for $\varepsilon_{2}=\left(\frac{1}{2 C_{6} M_{1} M_{2}^{r}}\right)^{1 / r}$ there exists $C_{8}>0$ such that

$$
g(t, u) \leq \varepsilon_{2} u^{1 / r}+C_{8}, \quad \forall(t, u) \in[0, T] \times[0, \infty)
$$


Hence, for $u \in P$, by using (22) and (23), we obtain

$$
\begin{aligned}
(A u)(t)= & \int_{0}^{T} G_{1}(t, s) f\left(s, \int_{0}^{T} G_{2}(s, \tau) g(\tau, u(\tau)) d \tau\right) d s \\
\leq & \int_{0}^{T} G_{1}(t, s)\left[C_{6}\left(\int_{0}^{T} G_{2}(s, \tau) g(\tau, u(\tau)) d \tau\right)^{r}+C_{7}\right] d s \\
\leq & C_{6} \int_{0}^{T} G_{1}(t, s)\left[\int_{0}^{T} G_{2}(s, \tau)\left(\varepsilon_{2}(u(\tau))^{1 / r}+C_{8}\right) d \tau\right]^{r} d s+M_{1} C_{7} \\
\leq & C_{6} \int_{0}^{T} G_{1}(t, s)\left[\int_{0}^{T} G_{2}(s, \tau)\left(\varepsilon_{2}\|u\|^{1 / r}+C_{8}\right) d \tau\right]^{r} d s+M_{1} C_{7} \\
= & C_{6}\left(\varepsilon_{2}\|u\|^{1 / r}+C_{8}\right)^{r}\left(\int_{0}^{T} I_{1}(s) d s\right)\left(\int_{0}^{T} I_{2}(\tau) d \tau\right)^{r}+M_{1} C_{7}, \\
& \forall t \in[0, T] .
\end{aligned}
$$

Therefore, we have

$$
(A u)(t) \leq C_{6} M_{1} M_{2}^{r}\left(\varepsilon_{2}\|u\|^{1 / r}+C_{8}\right)^{r}+M_{1} C_{7}, \quad \forall t \in[0, T] .
$$

After some computations, it can be shown that

$$
\lim _{\|u\| \rightarrow \infty}\left[C_{6} M_{1} M_{2}^{r}\left(\varepsilon_{2}\|u\|^{1 / r}+C_{8}\right)^{r}+M_{1} C_{7}\right] /\|u\|=\frac{1}{2},
$$

and so, there exists a sufficiently large $R>0$ such that

$$
C_{6} M_{1} M_{2}^{r}\left(\varepsilon_{2}\|u\|^{1 / r}+C_{8}\right)^{r}+M_{1} C_{7} \leq \frac{3}{4}\|u\|, \quad \forall u \in P, \quad\|u\| \geq R .
$$

Hence, from (24) and (25), we obtain $\|A u\| \leq \frac{3}{4}\|u\|<\|u\|$, for all $u \in$ $\partial B_{R} \cap P$, and from Theorem 2.1, we have

$$
i\left(A, B_{R} \cap P, P\right)=1 .
$$

On the other hand, from $(H 6)(\mathrm{i})$ we deduce that there exist positive constants $C_{9}>0$ and $\widetilde{u}_{1}>0$ such that $f(t, u) \geq C_{9} u, \forall(t, u) \in[0, T] \times\left[0, \widetilde{u}_{1}\right]$. From $(H 6)$ (ii), for $\varepsilon=C_{0} / C_{9}>0$ with $C_{0}=\frac{T^{n+m-2}}{c^{n+m-2} m_{1} m_{3}}>0$ and $m_{3}=$ $\int_{c}^{T-c} I_{2}(\tau) d \tau>0$, we conclude that there exists $\widetilde{\widetilde{u}}_{1}>0$ such that $g(t, u) \geq \frac{C_{0}}{C_{9}} u$ for all $(t, u) \in[0, T] \times\left[0, \widetilde{\widetilde{u}}_{1}\right]$. We consider $u_{1}=\min \left\{\widetilde{u}_{1}, \widetilde{\widetilde{u}}_{1}\right\}$ and then we obtain

$$
f(t, u) \geq C_{9} u, \quad g(t, u) \geq \frac{C_{0}}{C_{9}} u, \quad \forall(t, u) \in[0, T] \times\left[0, u_{1}\right] .
$$

Because $g(t, 0)=0$ for all $t \in[0, T]$, and $g$ is continuous, it can be shown that there exists a sufficiently small $\delta_{2} \in\left(0, u_{1}\right)$ such that $g(t, u) \leq \frac{u_{1}}{M_{2}}$ for all $(t, u) \in[0, T] \times\left[0, \delta_{2}\right]$. Hence,

$$
\begin{aligned}
& \int_{0}^{T} G_{2}(s, \tau) g(\tau, u(\tau)) d \tau \leq \int_{0}^{T} I_{2}(\tau) g(\tau, u(\tau)) d \tau \leq u_{1}, \quad \forall u \in \bar{B}_{\delta_{2}} \cap P \\
& s \in[0, T]
\end{aligned}
$$


From (27), (28) and Lemma 2.6, we deduce that for any $u \in \bar{B}_{\delta_{2}} \cap P$, we have

$$
\begin{aligned}
(A u)(t) & =\int_{0}^{T} G_{1}(t, s) f\left(s, \int_{0}^{T} G_{2}(s, \tau) g(\tau, u(\tau)) d \tau\right) d s \\
& \geq C_{9} \int_{0}^{T} G_{1}(t, s)\left(\int_{0}^{T} G_{2}(s, \tau) g(\tau, u(\tau)) d \tau\right) d s \\
& \geq C_{0} \int_{0}^{T} G_{1}(t, s)\left(\int_{0}^{T} G_{2}(s, \tau) u(\tau) d \tau\right) d s \\
& \geq C_{0} \int_{c}^{T-c} G_{1}(t, s)\left(\int_{0}^{T} G_{2}(s, \tau) u(\tau) d \tau\right) d s \\
& \geq \frac{C_{0} c^{m-1}}{T^{m-1}} \int_{c}^{T-c} G_{1}(t, s)\left(\int_{0}^{T} I_{2}(\tau) u(\tau) d \tau\right) d s=(L u)(t), \quad \forall t \in[0, T]
\end{aligned}
$$

where the linear operator $L: P \rightarrow P$ is defined by

$$
(L u)(t)=\frac{C_{0} c^{m-1}}{T^{m-1}}\left(\int_{0}^{T} I_{2}(\tau) u(\tau) d \tau\right)\left(\int_{c}^{T-c} G_{1}(t, s) d s\right), \quad t \in[0, T] .
$$

Hence, we obtain

$$
A u \geq L u, \quad \forall u \in \partial B_{\delta_{2}} \cap P .
$$

For $w_{0}(t)=\int_{c}^{T-c} G_{1}(t, s) d s, t \in[0, T]$, we have $w_{0} \in P \backslash\left\{\theta_{0}\right\}$ and

$$
\begin{aligned}
\left(L w_{0}\right)(t) & =\frac{C_{0} c^{m-1}}{T^{m-1}}\left[\int_{0}^{T} I_{2}(\tau)\left(\int_{c}^{T-c} G_{1}(\tau, s) d s\right) d \tau\right]\left(\int_{c}^{T-c} G_{1}(t, s) d s\right) \\
& \geq \frac{C_{0} c^{n+m-2}}{T^{n+m-2}}\left(\int_{c}^{T-c} I_{2}(\tau) d \tau\right)\left(\int_{c}^{T-c} I_{1}(\tau) d \tau\right)\left(\int_{c}^{T-c} G_{1}(t, s) d s\right) \\
& =\frac{C_{0} c^{n+m-2} m_{1} m_{3}}{T^{n+m-2}} \int_{c}^{T-c} G_{1}(t, s) d s=\int_{c}^{T-c} G_{1}(t, s) d s=w_{0}(t), \quad t \in[0, T] .
\end{aligned}
$$

Therefore,

$$
L w_{0} \geq w_{0} .
$$

We may suppose that $A$ has no fixed point on $\partial B_{\delta_{2}} \cap P$ (otherwise the proof is finished). From (29), (30) and Theorem 2.3 (with $u_{0}=w_{0}$ ), we conclude that

$$
i\left(A, B_{\delta_{2}} \cap P, P\right)=0 .
$$

Therefore, from (26) and (31), we have

$$
i\left(A,\left(B_{R} \backslash \bar{B}_{\delta_{2}}\right) \cap P, P\right)=i\left(A, B_{R} \cap P, P\right)-i\left(A, B_{\delta_{2}} \cap P, P\right)=1 .
$$

Then $A$ has at least one fixed point in $\left(B_{R} \backslash \bar{B}_{\delta_{2}}\right) \cap P$. Thus, the problem $(S)-(B C)$ has at least one positive solution $(u, v) \in P \times P$ with $u(t) \geq$ $0, v(t) \geq 0$ for all $t \in[0, T]$ and $\|u\|>0,\|v\|>0$. This completes the proof of Theorem 3.2. 
Theorem 3.3. Assume that $(H 1)-(H 3),(H 6)$ and $(H 7)$ hold. Then the problem $(S)-(B C)$ has at least two positive solutions $\left(u_{1}(t), v_{1}(t)\right),\left(u_{2}(t), v_{2}(t)\right), t \in$ $[0, T]$.

Proof. From Sect. 2, we have $0 \leq G_{1}(t, s) \leq I_{1}(s) \leq K_{1}$ and $G_{2}(t, s) \leq I_{2}(s) \leq$ $K_{2}$, for all $(t, s) \in[0, T] \times[0, T]$. By using $(H 7)$, for any $u \in \partial B_{N} \cap P$, we obtain

$$
\begin{aligned}
(A u)(t) & =\int_{0}^{T} G_{1}(t, s) f\left(s, \int_{0}^{T} G_{2}(s, \tau) g(\tau, u(\tau)) d \tau\right) d s \\
& \leq \int_{0}^{T} G_{1}(t, s) f\left(s, K_{2} \int_{0}^{T} g(\tau, u(\tau)) d \tau\right) d s \\
& \leq \int_{0}^{T} G_{1}(t, s) f\left(s, m_{0} \int_{0}^{T} g(\tau, N) d \tau\right) d s \\
& <\frac{N}{m_{0}} \int_{0}^{T} G_{1}(t, s) d s \leq \frac{N}{m_{0}} K_{1} T \leq N, \quad \forall t \in[0, T] .
\end{aligned}
$$

So, $\|A u\|<\|u\|$ for all $u \in \partial B_{N} \cap P$.

By Theorem 2.1, we conclude that

$$
i\left(A, B_{N} \cap P, P\right)=1 .
$$

On the other hand, from $(H 3),(H 6)$ and the proofs of Theorems 3.1 and 3.2 , we know that there exists a sufficiently large $L>N$ and a sufficiently small $\delta_{2}$ with $0<\delta_{2}<N$ such that

$$
i\left(A, B_{L} \cap P, P\right)=0, \quad i\left(A, B_{\delta_{2}} \cap P, P\right)=0 .
$$

From the relations (32) and (33), we obtain

$$
\begin{aligned}
& i\left(A,\left(B_{L} \backslash \bar{B}_{N}\right) \cap P, P\right)=i\left(A, B_{L} \cap P, P\right)-i\left(A, B_{N} \cap P, P\right)=-1 \\
& i\left(A,\left(B_{N} \backslash \bar{B}_{\delta_{2}}\right) \cap P, P\right)=i\left(A, B_{N} \cap P, P\right)-i\left(A, B_{\delta_{2}} \cap P, P\right)=1 .
\end{aligned}
$$

Then $A$ has at least one fixed point $u_{1}$ in $\left(B_{L} \backslash \bar{B}_{N}\right) \cap P$ and has one fixed point $u_{2}$ in $\left(B_{N} \backslash \bar{B}_{\delta_{2}}\right) \cap P$, respectively. Therefore, the problem $(S)-(B C)$ has two distinct positive solutions $\left(u_{1}, v_{1}\right),\left(u_{2}, v_{2}\right) \in P \times P$ with $u_{i}(t) \geq 0, v_{i}(t) \geq 0$ for all $t \in[0, T]$ and $\left\|u_{i}\right\|>0,\left\|v_{i}\right\|>0, i=1,2$. The proof of Theorem 3.3 is completed.

\section{Examples}

In this section, we shall present some examples which illustrate our results.

Example 1. Let $f(t, u)=\left(a t^{\alpha}+b\right) u^{1 / 2}(2+\sin u)$ and $g(t, u)=\left(c t^{\beta}+d\right) u^{3}(3+$ $\cos u$ ) with $a, c \geq 0, b, d>0, \alpha, \beta>0$ and $p_{1}=1 / 2, q_{1}=1 / 2$. Then the 
assumptions (H3) and (H4) are satisfied; indeed, we have

$$
\begin{aligned}
f_{\infty}^{i} & =\liminf _{u \rightarrow \infty} \inf _{t \in[0, T]} \frac{\left(a t^{\alpha}+b\right) u^{1 / 2}(2+\sin u)}{u^{1 / 2}}=b, \\
g_{\infty}^{i} & =\liminf _{u \rightarrow \infty} \inf _{t \in[0, T]} \frac{\left(c t^{\beta}+d\right) u^{3}(3+\cos u)}{u^{2}}=\infty, \\
f_{0}^{s} & =\limsup _{u \rightarrow 0^{+}} \sup _{t \in[0, T]} \frac{\left(a t^{\alpha}+b\right) u^{1 / 2}(2+\sin u)}{u^{1 / 2}}=2\left(a T^{\alpha}+b\right), \\
g_{0}^{s} & =\limsup _{u \rightarrow 0^{+}} \sup _{t \in[0, T]} \frac{\left(c t^{\beta}+d\right) u^{3}(3+\cos u)}{u^{2}}=0 .
\end{aligned}
$$

Under the assumption $(H 1)$, by Theorem 3.1, we deduce that the problem $(S)-(B C)$ has at least one positive solution.

Example 2. Let $f(t, u)=u^{1 / 2}, g(t, u)=u^{1 / 3}$ and $r=1 / 2$. Then the assumptions (H5) and (H6) are satisfied; indeed, we have

$$
\begin{aligned}
f_{\infty}^{s} & =\lim _{u \rightarrow \infty} \frac{u^{1 / 2}}{u^{1 / 2}}=1, \quad g_{\infty}^{s}=\lim _{u \rightarrow \infty} \frac{u^{1 / 3}}{u^{2}}=0, \quad f_{0}^{i}=\lim _{u \rightarrow 0^{+}} \frac{u^{1 / 2}}{u}=\infty \\
g_{0}^{i} & =\lim _{u \rightarrow 0^{+}} \frac{u^{1 / 3}}{u}=\infty
\end{aligned}
$$

Under the assumption $(H 1)$, by Theorem 3.2, we conclude that the problem $(S)-(B C)$ has at least one positive solution.

Example 3. We consider the following problem

$$
\left\{\begin{array}{l}
u^{(3)}(t)+a\left(v^{\alpha}+v^{\beta}\right)=0, \quad t \in(0,1) \\
v^{(4)}(t)+b\left(u^{\gamma}+u^{\delta}\right)=0, \quad t \in(0,1)
\end{array}\right.
$$

with the multi-point boundary conditions

$$
\left\{\begin{array}{l}
u(0)=u^{\prime}(0)=0, \quad u(1)=2 u\left(\frac{1}{4}\right)+u\left(\frac{1}{2}\right)+\frac{1}{2} u\left(\frac{3}{4}\right), \\
v(0)=v^{\prime}(0)=v^{\prime \prime}(0)=0, \quad v(1)=v\left(\frac{1}{3}\right)+3 v\left(\frac{2}{3}\right),
\end{array} \quad\left(B C_{0}\right)\right.
$$

where $\alpha>1, \beta<1, \gamma>2, \delta<1, a, b>0$. Here $T=1, n=3, m=4, p=5, q=$ $4, \xi_{1}=\frac{1}{4}, \xi_{2}=\frac{1}{2}, \xi_{3}=\frac{3}{4}, a_{1}=2, a_{2}=1, a_{3}=\frac{1}{2}, \eta_{1}=\frac{1}{3}, \eta_{2}=\frac{2}{3}, b_{1}=1, b_{2}=3$. Then, we have $d=1-\sum_{i=1}^{3} a_{i} \xi_{i}^{2}=\frac{11}{32}>0, e=1-\sum_{i=1}^{3} b_{i} \eta_{i}^{3}=\frac{2}{27}>0$. Here,

$$
\theta_{1}(s)=\frac{1}{2-s}, \quad s \in[0,1] ; \quad \theta_{2}(s)= \begin{cases}\frac{s}{1-(1-s)^{3 / 2}}, & s \in(0,1] \\ \frac{2}{3}, \quad s=0 & \end{cases}
$$


For the functions $I_{1}$ and $I_{2}$, we obtain

$$
\begin{aligned}
& I_{1}(s)=g_{1}\left(\theta_{1}(s), s\right)+\frac{1}{d}\left[2 g_{1}\left(\frac{1}{4}, s\right)+g_{1}\left(\frac{1}{2}, s\right)+\frac{1}{2} g_{1}\left(\frac{3}{4}, s\right)\right] \\
& = \begin{cases}\frac{2 s-5 s^{2}+4 s^{3}-s^{4}}{2(2-s)^{2}}+\frac{1}{22}\left(-91 s^{2}+46 s\right), & 0 \leq s<\frac{1}{4}, \\
\frac{2 s-5 s^{2}+4 s^{3}-s^{4}}{2(2-s)^{2}}+\frac{1}{22}\left(-27 s^{2}+14 s+4\right), & \frac{1}{4} \leq s<\frac{1}{2}, \\
\frac{2 s-5 s^{2}+4 s^{3}-s^{4}}{2(2-s)^{2}}+\frac{1}{22}\left(5 s^{2}-18 s+12\right), & \frac{1}{2} \leq s<\frac{3}{4}, \\
\frac{2 s-5 s^{2}+4 s^{3}-s^{4}}{2(2-s)^{2}}+\frac{21}{22}\left(1-2 s+s^{2}\right), & \frac{3}{4} \leq s \leq 1,\end{cases} \\
& I_{2}(s)=g_{2}\left(\theta_{2}(s), s\right)+\frac{1}{e}\left[g_{2}\left(\frac{1}{3}, s\right)+3 g_{2}\left(\frac{2}{3}, s\right)\right] \\
& = \begin{cases}0, & s=0, \\
\frac{s^{3}(1-s)^{3}-s^{3}(1-s)^{9 / 2}}{6\left[1-(1-s)^{3 / 2}\right]^{3}}+\frac{1}{12}\left(83 s^{3}-114 s^{2}+42 s\right), & 0<s<\frac{1}{3}, \\
\frac{s^{3}(1-s)^{3}-s^{3}(1-s)^{9 / 2}}{6\left[1-(1-s)^{3 / 2}\right]^{3}}+\frac{1}{12}\left(56 s^{3}-87 s^{2}+33 s+1\right), & \frac{1}{3} \leq s<\frac{2}{3}, \\
\frac{s^{3}(1-s)^{3}-s^{3}(1-s)^{9 / 2}}{6\left[1-(1-s)^{3 / 2}\right]^{3}}+\frac{25}{12}\left(1-3 s+3 s^{2}-s^{3}\right), & \frac{2}{3} \leq s \leq 1 .\end{cases}
\end{aligned}
$$

We have $K_{1}=\max _{s \in[0,1]} I_{1}(s) \approx 0.306, K_{2}=\max _{s \in[0,1]} I_{2}(s) \approx 0.398$. Then $m_{0}=\max \left\{K_{1} T, K_{2}\right\}=K_{2}$. The functions $f(t, u)$ and $g(t, u)$ are nondecreasing with respect to $u$, for any $t \in[0,1]$, and for $p_{1}=1 / 2$ the assumptions (H3) and $(H 6)$ are satisfied; indeed we obtain

$$
\begin{aligned}
& f_{\infty}^{i}=\lim _{u \rightarrow \infty} \frac{a\left(u^{\alpha}+u^{\beta}\right)}{u^{1 / 2}}=\infty, \quad g_{\infty}^{i}=\lim _{u \rightarrow \infty} \frac{b\left(u^{\gamma}+u^{\delta}\right)}{u^{2}}=\infty, \\
& f_{0}^{i}=\lim _{u \rightarrow 0^{+}} \frac{a\left(u^{\alpha}+u^{\beta}\right)}{u}=\infty, \quad g_{0}^{i}=\lim _{u \rightarrow 0^{+}} \frac{b\left(u^{\gamma}+u^{\delta}\right)}{u}=\infty .
\end{aligned}
$$

We take $N=1$ and then $\int_{0}^{1} g(s, 1) d s=2 b$ and $f\left(t, 2 b m_{0}\right)=a\left[\left(2 b m_{0}\right)^{\alpha}+\right.$ $\left.\left(2 b m_{0}\right)^{\beta}\right]$. If $a\left[\left(2 b m_{0}\right)^{\alpha}+\left(2 b m_{0}\right)^{\beta}\right]<\frac{1}{m_{0}} \Leftrightarrow a\left[m_{0}^{\alpha+1}(2 b)^{\alpha}+m_{0}^{\beta+1}(2 b)^{\beta}\right]<1$, then the assumption (H7) is satisfied. For example, if $\alpha=2, \beta=1 / 2, b=1 / 2$ and $a<\frac{1}{m_{0}^{3}+m_{0}^{3 / 2}}$ (e.g. $a \leq 3$ ), then the above inequality is satisfied. By Theorem 3.3 , we deduce that the problem $\left(S_{0}\right)-\left(B C_{0}\right)$ has at least two positive solutions.

\section{Acknowledgments}

The authors thank the referee for his/her valuable comments and suggestions. The work of R. Luca was supported by the CNCS Grant PN-II-ID-PCE-20113-0557, Romania. 


\section{References}

[1] Amann, H.: Fixed point equations and nonlinear eigenvalue problems in ordered Banach spaces. SIAM Rev. 18, 620-709 (1976)

[2] Eloe, P.W., Henderson, J.: Positive solutions for $(n-1,1)$ conjugate boundary value problems. Nonlinear Anal. 28, 1669-1680 (1997)

[3] Henderson, J., Luca, R.: Positive solutions for a system of higher-order multipoint boundary value problems. Comput. Math. Appl. 62, 3920-3932 (2011)

[4] Henderson, J., Luca, R.: Positive solutions for a system of second-order multipoint boundary value problems. Appl. Math. Comput. 218, 6083-6094 (2012)

[5] Henderson, J., Luca, R.: Existence and multiplicity for positive solutions of a multi-point boundary value problem. Appl. Math. Comput. 218, 10572$10585(2012)$

[6] Henderson, J., Ntouyas, S.K.: Positive solutions for systems of $n$th order threepoint nonlocal boundary value problems. Electron. J. Qual. Theory Differ. Equ. 18, 1-12 (2007)

[7] Henderson, J., Ntouyas, S.K.: Positive solutions for systems of three-point nonlinear boundary value problems. Aust. J. Math. Anal. Appl. 5, 1-9 (2008)

[8] Henderson, J., Ntouyas, S.K., Purnaras, I.K.: Positive solutions for systems of generalized three-point nonlinear boundary value problems. Comment. Math. Univ. Carol. 49, 79-91 (2008)

[9] Henderson, J., Ntouyas, S.K., Purnaras, I.K.: Positive solutions for systems of $m$-point nonlinear boundary value problems Math. Model. Anal. 13, 357-370 (2008)

[10] Ji, Y., Guo, Y.: The existence of countably many positive solutions for some nonlinear $n$th order $m$-point boundary value problems. J. Comput. Appl. Math. 232, 187-200 (2009)

[11] Ji, Y., Guo, Y., Yu, C.: Positive solutions to $(n-1, n) m$-point boundary value problems with dependence on the first order derivative. Appl. Math. Mech. (Engl. Ed.) 30, 527-536 (2009)

[12] Luca, R.: Existence of positive solutions for a class of higher-order $m$-point boundary value problems. Electron. J. Qual. Theory Diff. Equ. 74, 1-15 (2010)

[13] Luca, R.: Positive solutions for a second-order $m$-point boundary value problem. Dyn. Contin. Discrete Impuls. Syst. Ser. A Math. Anal. 18, 161-176 (2011)

[14] Su, H., Wei, Z., Zhang, X., Liu, J.: Positive solutions of $n$-order and $m$-order multi-point singular boundary value system. Appl. Math. Comput. 188, 1234$1243(2007)$ 
[15] Sun, H.R., Li, W.T.: Existence of positive solutions for nonlinear three-point boundary value problems on time scales. J. Math. Anal. Appl. 299, 508524 (2004)

[16] Zhou, Y., Xu, Y.: Positive solutions of three-point boundary value problems for systems of nonlinear second order ordinary differential equations. J. Math. Anal. Appl. 320, 578-590 (2006)

\section{J. Henderson}

Department of Mathematics

Baylor University

Waco

TX 76798-7328

USA

e-mail: Johnny_Henderson@baylor.edu

R. Luca

Department of Mathematics

Gh. Asachi Technical University

700506 Iasi

Romania

e-mail: rlucatudor@yahoo.com;

rluca@math.tuiasi.ro

Received: 25 April 2012.

Accepted: 4 August 2012. 